\title{
On shrinkage estimators improving the James-Stein estimator under balanced loss function
}

\author{
Abdenour Hamdaoui ${ }^{1 *}$, Mekki Terbeche ${ }^{2}$, \\ Abdelkader Benkhaled ${ }^{3}$ \\ ${ }^{*}$ Corresponding author
}

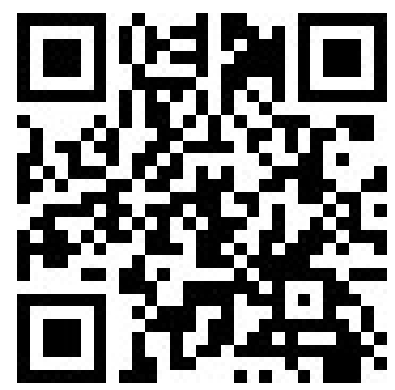

1. Department of Mathematics, University of Sciences and Technology, Mohamed Boudiaf, Oran, Laboratory of Statistics and Random Modelisations of University Abou Bekr Belkaid (LSMA), Tlemcen

\& El Mnaouar, BP 1505, Bir El Djir 31000, Oran, Algeria. E-mail: abdenour.hamdaoui@yahoo.fr

2. Department of Mathematics, University of Sciences and Technology, Mohamed Boudiaf, Oran, Laboratory of Analysis and Application of Radiation (LAAR), USTO-MB, Algeria. E-mail:mekki.terbeche@gmail.com

3. Department of Biology, University of Mascara, Laboratory of Geomatics, Ecology and Environment (LGEO2E) of Mascara University, Algeria. E-mail: benkhaled08@yahoo.fr

\begin{abstract}
In this paper, we are interested in estimating a multivariate normal mean under the balanced loss function using the shrinkage estimators deduced from the Maximum Likelihood Estimator (MLE). First, we consider a class of estimators containing the James-Stein estimator, we then show that any estimator of this class dominates the MLE, consequently it is minimax. Secondly, we deal with shrinkage estimators which are not only minimax but also dominate the JamesStein estimator.
\end{abstract}

Key Words: Balanced loss function; James-Stein estimator; minimax estimator; non-central chi-square distribution; shrinkage estimators

Mathematical Subject Classification: Primary 62C20. Secondary 62H10.

\section{Introduction}

The problem of estimation the mean parameters is the most widely used statistical tool applied in almost all fields. This problem has been attracted the attention of many researchers for multivariate normal distribution. Several deterministic approaches have been proposed in this context. Stein(1956) discovered that when the dimension of the parameter space is large, the usual maximum likelihood estimator is inadmissible. James and Stein(1961) provided an explicit class of dominating estimators. They provided results for both cases of known and unknown variance. Alternative techniques have been developed to improve the MLE. In this paper, we aim to develop shrinkage estimators that are both minimax and capable of effective risk reduction over the usual estimator. Recent studies, in the context of shrinkage estimation, include Selahattin et al.(2011), Amin et al.(2020), Yuzba et al.(2020). Tsukuma and Kubukawa(2015) address the problem of estimating the mean vector of a singular multivariate normal distribution with an unknown singular covariance matrix. Xie et al.(2016) introduced a class of semi-parametric/parametric shrinkage estimators and established their asymptotic optimality properties. Benkhaled and Hamdaoui(2019), have considered the model $X \sim N_{p}\left(\theta, \sigma^{2} I_{p}\right)$ where $\sigma^{2}$ is unknown and studied two different forms of shrinkage estimators of $\theta$ : estimators of the form $\delta^{\psi}=\left(1-\psi\left(S^{2},\|X\|^{2}\right) S^{2} /\|X\|^{2}\right) X$, and estimators of Lindley-Type given by 
$\delta^{\varphi}=\left(1-\varphi\left(S^{2}, T^{2}\right) S^{2} / T^{2}\right)(X-\bar{X})+\bar{X}$, that shrink the components of the MLE $X$ to the random variable $\bar{X}$. The authors showed that if the shrinkage function $\psi$ (respectively $\varphi$ ) satisfies the new conditions different from the known results in the literature, then the estimator $\delta^{\psi}$ (respectively $\delta^{\varphi}$ ) is minimax. When both sample size and the dimension of parameters space tend to infinity, they studied the behaviour of risks ratio of these estimators to the MLE. Hamdaoui et al.(2020), have treated the minimaxity and limits of risks ratios of shrinkage estimators of a multivariate normal mean in the Bayesian case. The authors have considered the model $X \sim N_{p}\left(\theta, \sigma^{2} I_{p}\right)$ where $\sigma^{2}$ is unknown and have taken the prior law $\theta \sim N_{p}\left(v, \tau^{2} I_{p}\right)$. They constructed a modified Bayes estimator $\delta_{B}^{*}$ and an empirical modified Bayes estimator $\delta_{E B}^{*}$. When $n$ and $p$ are finite, they showed that the estimators $\delta_{B}^{*}$ and $\delta_{E B}^{*}$ are minimax. The authors have also interested in studying the limits of risks ratios of these estimators, to the MLE $X$, when $n$ and $p$ tend to infinity. The majority of these authors have been considered the quadratic loss function for computing the risk.

Zellner(1994) proposed a balanced loss function that takes error of estimation and goodness of fit into account. This balanced loss function consists of weighting the predictive loss function and the goodness of fit term. Sanjari and Asgharzadeh(2004) have considered the model: $X_{1}, \ldots, X_{n}$ to be a random sample from $N_{p}\left(\theta, \sigma^{2} I_{p}\right)$ with $\sigma^{2}$ known and the aim is to estimate the parameter $\theta$. They studied the admissibility of the estimator of the form $a \bar{X}+b$ under the balanced loss function. Selahattin and Issam(2019) introduced and derived the optimal extended balanced loss function (EBLF) estimators and predictors and discussed their performances.

In this work, we deal with the model $X \sim N_{p}\left(\theta, \sigma^{2} I_{p}\right)$, where the parameter $\sigma^{2}$ is known. Our aim is to estimate the unknown parameter $\theta$ by shrinkage estimators deduced from the MLE. The adopted criterion to compare two estimators is the risk associated to the balanced loss function. The rest of this article is organized as follows. Section 2 contains some necessary preliminaries that are useful for our main results. In the first part of the Section 3, we establish the minimaxity of the estimators defined by $\delta_{a, r}=\left(1-a\left(1 /\|X\|^{r}\right)\right) X$, where $2 \leq r<(p+2) / 2$ and the real constant $a$ may depend on $p$. In the second part of the Section 3, we consider the estimators of the form $\delta_{b, r}=\delta_{J S}+b\left(1 /\|X\|^{r}\right) X$ with $2 \leq r<(p+2) / 2$ and the real constant $b$ may depend on $p$. We show that these estimators dominate the James-Stein estimator $\delta_{J S}$ under some conditions on the parameter $b$. Section 4 is devoted to some numerical comparisons. The proofs of some our main results are collected in the Appendix.

\section{Preliminaries}

We recall that if $X$ is a multivariate Gaussian random $N_{p}\left(\theta, \sigma^{2} I_{p}\right)$ in $\mathrm{R}^{p}$, then $\frac{\|X\|^{2}}{\sigma^{2}} \sim \chi_{p}^{2}(\lambda)$ where $\chi_{p}^{2}(\lambda)$ denotes the non-central chi-square distribution with $p$ degrees of freedom and non-centrality parameter $\lambda=\frac{\|\theta\|^{2}}{2 \sigma^{2}}$. We also recall the following definition given in formula (1.2) by Arnold(1981). It will be used to calculate the expectation of functions of a non-central chi-square law's variable.

Definition 2.1. Let $U \sim \chi_{p}^{2}(\lambda)$ be non-central chi-square with $p$ degrees of freedom and non-centrality parameter $\lambda$. The density function of $U$ is given by

$$
f(x)=\sum_{k=0}^{+\infty} \frac{e^{-\frac{\lambda}{2}}\left(\frac{\lambda}{2}\right)^{k}}{k !} \frac{x^{(p / 2)+k-1} e^{-x / 2}}{\Gamma\left(\frac{p}{2}+k\right) 2^{(p / 2)+k}}, 0<x<+\infty .
$$

The right hand side (RHS) of this equality is none other than the formula

$$
\sum_{k=0}^{+\infty} \frac{e^{-\frac{\lambda}{2}}\left(\frac{\lambda}{2}\right)^{k}}{k !} \chi_{p+2 k}^{2},
$$

where $\chi_{p+2 k}^{2}$ is the density of the central $\chi^{2}$ distribution with $p+2 k$ degrees of freedom.

To this definition we deduce that if $U \sim \chi_{p}^{2}(\lambda)$, then for any function $f: \mathrm{R}_{+} \longrightarrow \mathrm{R}, \chi_{p}^{2}(\lambda)$ integrable, we have

$$
E[f(U)]=\int_{\mathrm{R}_{+}} f(x) \chi_{p}^{2}(\lambda) d x
$$




$$
\begin{aligned}
& =\sum_{k=0}^{+\infty}\left[\int_{\mathrm{R}_{+}} f(x) \chi_{p+2 k}^{2}(0) d x\right] e^{-\frac{\lambda}{2}} \frac{\left(\frac{\lambda}{2}\right)^{k}}{k !} \\
& =\sum_{k=0}^{+\infty}\left[\int_{\mathrm{R}_{+}} f(x) \chi_{p+2 k}^{2} d x\right] P\left(\frac{\lambda}{2} ; d k\right),
\end{aligned}
$$

where $P\left(\frac{\lambda}{2} ; d k\right)$ being the Poisson distribution of parameter $\frac{\lambda}{2}$ and $\chi_{p+2 k}^{2}$ is the central chi-square distribution with $p+2 k$ degrees of freedom.

Using the last equality, we conclude the following Lemma.

Proposition 2.1. Let $U \sim \chi_{p}^{2}(\lambda)$ be non-central chi-square with $p$ degrees of freedom and non-centrality parameter $\lambda$. Then for $0 \leq r<\frac{p}{2}$,

$$
\begin{aligned}
E\left(U^{-r}\right) & =E\left[\left(\chi_{p}^{2}(\lambda)\right)^{-r}\right] \\
& =E\left[\left(\chi_{p+2 K}^{2}\right)^{-r}\right] \\
& =2^{-r} E\left(\frac{\Gamma\left(\frac{p}{2}-r+K\right)}{\Gamma\left(\frac{p}{2}+K\right)}\right),
\end{aligned}
$$

where K has a Poisson distribution with mean $\frac{\lambda}{2}$.

We recall the following Lemma given by Stein (13), that we will use often in the next.

Proposition 2.2. Let $X$ be a $N\left(v, \sigma^{2}\right)$ real random variable and let $f: \mathrm{R} \longrightarrow \mathrm{R}$ be an indefinite integral of the Lebesgue measurable function, $f^{\prime}$ essentially the derivative of $f$. Suppose also that $E\left(\left|f^{\prime}(X)\right|\right)<+\infty$, then

$$
E\left[\left(\frac{X-v}{\sigma}\right) f(X)\right]=E\left(f^{\prime}(X)\right) .
$$

\section{Main results}

In this section, we present the model $X \sim N_{p}\left(\theta, \sigma^{2} I_{p}\right)$ where $\sigma^{2}$ is known. Our aim is to estimate the unknown mean parameter $\theta$ by the shrinkage estimators under the balanced squared error loss function. For the sake of simplicity, we treat only the case when $\sigma^{2}=1$, as long as by a change of variable, any model of type $Y \sim N_{p}\left(\theta_{1}, \sigma^{2} I_{p}\right)$ can be reduced to the model $Z \sim N_{p}\left(\theta_{2}, I_{p}\right)$. Namely, we consider the model $X \sim N_{p}\left(\theta, I_{p}\right)$ and we want to estimate the unknown parameter $\theta$.

Definition 3.1. Suppose that $X$ is a random vector having a multivariate normal distribution $N_{p}\left(\theta, I_{p}\right)$ where the parameter $\theta$ is unknown. The balanced squared error loss function is defined as follows:

$$
L_{\omega}(\delta, \theta)=\omega\left\|\delta-\delta_{0}\right\|^{2}+(1-\omega)\|\delta-\theta\|^{2}, 0 \leq \omega<1,
$$

where $\delta_{0}$ is the target estimator of $\theta, \omega$ is the weight given to the proximity of $\delta$ to $\delta_{0}, 1-\omega$ is the relative weight given to the precision of estimation portion and $\delta$ is a given estimator.

For more details about this loss see Jafari et al.(2014), Guikai et al.(2014), Zinodiny et al.(2017) and Karamikabir et al.(2018).

We associate to this balanced squared error loss function the risk function defined by

$$
R_{\omega}(\delta, \theta)=E\left(L_{\omega}(\delta, \theta)\right) .
$$

In this model, it is clear that the MLE is $X:=\delta_{0}$, its risk function is $(1-\omega) p$.

Indeed: we have

$$
\begin{aligned}
R_{\omega}(X, \theta) & =\omega E\left(\|X-X\|^{2}\right)+(1-\omega) E\left(\|X-\theta\|^{2}\right) \\
& =(1-\omega) E\left(\|X-\theta\|^{2}\right) .
\end{aligned}
$$


As $X \sim N_{p}\left(\theta, I_{p}\right)$, then $X-\theta \sim N_{p}\left(0, I_{p}\right)$, therefore $\|X-\theta\|^{2} \sim \chi_{p}^{2}$.

Hence, $E\left(\|X-\theta\|^{2}\right)=E\left(\chi_{p}^{2}\right)=p$, and the desired result follows.

It is well known that $\delta_{0}$ is minimax and inadmissible for $p \geq 3$, thus any estimator dominates it is also minimax. We give the following Lemma, that will be used in our proofs and its proof is postponed to the Appendix.

Proposition 3.1. Let $U \sim \chi_{p}^{2}(\lambda)$ be non-central chi-square with $p$ degrees of freedom and non-centrality parameter $\lambda$ then,

i) for any real numbers $s$ and $r$ where $-\frac{p}{2}<s \leq r<0$, the real function

$$
H_{p, r, s}(\lambda)=\frac{E\left(U^{r}\right)}{E\left(U^{s}\right)}=\frac{\int_{R_{+}} x^{r} \chi_{p}^{2}(\lambda ; d x)}{\int_{R_{+}} x^{s} \chi_{p}^{2}(\lambda ; d x)}
$$

is nondecreasing on $\lambda$.

ii) Furthermore, if $X \sim N_{p}\left(\theta, I_{p}\right)$, we get

$$
\sup _{\|\theta\|}\left(\frac{E\left(\|X\|^{-2 r+2}\right)}{E\left(\|X\|^{-r}\right)}\right)=2^{\frac{-r+2}{2}} \frac{\Gamma\left(\frac{p}{2}-r+1\right)}{\Gamma\left(\frac{p-r}{2}\right)} .
$$

\subsection{A general class of James-Stein estimator}

Consider the estimator

$$
\delta_{a, r}=\left(1-a \frac{1}{\|X\|^{r}}\right) X=X-a \frac{1}{\|X\|^{r}} X
$$

where $2 \leq r<\frac{p+2}{2}$ and the real positive constant $a$ may depend on $p$.

Proposition 3.1. Under the balanced squared error loss function $L_{\omega}$, the risk function of the estimator $\delta_{a, r}$ given in (3) is

$$
R_{\omega}\left(\delta_{a, r}, \theta\right)=(1-\omega)\left\{p-2 a(p-r) E\left(\frac{1}{\|X\|^{r}}\right)\right\}+a^{2} E\left(\frac{1}{\|X\|^{2 r-2}}\right)
$$

Proof. Using the risk function associated to the balanced squared error loss function defined in (2), we obtain

$$
R_{\omega}\left(\delta_{a, r}, \theta\right)=\omega E\left(\left\|\delta_{a, r}-X\right\|^{2}\right)+(1-\omega) E\left(\left\|\delta_{a, r}-\theta\right\|^{2}\right) .
$$

As,

$$
\begin{aligned}
E\left(\left\|\delta_{a, r}-X\right\|^{2}\right)=E\left(\left\|-a \frac{1}{\|X\|^{r}} X\right\|^{2}\right)=a^{2} E\left(\frac{1}{\|X\|^{2 r-2}}\right), & \\
E\left(\left\|\delta_{a, r}-\theta\right\|^{2}\right) & =E\left(\left\|X-a \frac{1}{\|X\|^{r}} X-\theta\right\|^{2}\right) \\
& =E\left(\|X-\theta\|^{2}\right)+E\left(\left\|a \frac{1}{\|X\|^{r}} X\right\|^{2}\right)-2 E\left(\left\langle X-\theta, a \frac{1}{\|X\|^{r}} X\right\rangle\right)
\end{aligned}
$$


and using the Lemma 2.2, we get

$$
\begin{aligned}
E\left(\left\langle X-\theta, a \frac{1}{\|X\|^{r}} X\right\rangle\right) & =a \sum_{i=1}^{p} E\left[\left(X_{i}-\theta_{i}\right) \frac{1}{\|X\|^{r}} X_{i}\right] \\
& =a \sum_{i=1}^{p} E\left(\frac{\partial}{\partial X_{i}} \frac{1}{\|X\|^{r}} X_{i}\right) \\
& =a \sum_{i=1}^{p} E\left(\frac{1}{\|X\|^{r}}-\frac{r X_{i}^{2}}{\|X\|^{r+2}}\right) \\
& =a(p-r) E\left(\frac{1}{\|X\|^{r}}\right) .
\end{aligned}
$$

Then

$$
\begin{aligned}
R_{\omega}\left(\delta_{a, r}, \theta\right) & =\omega a^{2} E\left(\frac{1}{\|X\|^{2 r-2}}\right)+(1-\omega)\left[p+a^{2} E\left(\frac{1}{\|X\|^{2 r-2}}\right)-2 a(p-r) E\left(\frac{1}{\|y\|^{r}}\right)\right] \\
& =(1-\omega)\left\{p-2 a(p-r) E\left(\frac{1}{\|X\|^{r}}\right)\right\}+a^{2} E\left(\frac{1}{\|X\|^{2 r-2}}\right),
\end{aligned}
$$

and the desired result is obtained.

Theorem 3.2. Assume the estimator $\delta_{a, r}$ defined in (3).

i) A sufficient condition that $\delta_{a, r}$ dominates the MLE (so it is minimax), is

$$
0 \leq a \leq 2^{\frac{r}{2}}(1-\omega)(p-r) \frac{\Gamma\left(\frac{p-r}{2}\right)}{\Gamma\left(\frac{p-2 r+2}{2}\right)},
$$

ii) the optimal value for a that minimizes the risk function $R_{\omega}\left(\delta_{a, r}, \theta\right)$, is

$$
\widehat{a}=2^{\frac{r-2}{2}}(1-\omega)(p-r) \frac{\Gamma\left(\frac{p-r}{2}\right)}{\Gamma\left(\frac{p-2 r+2}{2}\right)} .
$$

Proof. i) By using Proposition 3.1, we have

$$
\begin{aligned}
R_{\omega}\left(\delta_{a, r}, \theta\right) & =(1-\omega)\left\{p-2 a(p-r) E\left(\frac{1}{\|X\|^{r}}\right)\right\} \\
& +a^{2}\left(\frac{E\left(\frac{1}{\|X\|^{2 r-2}}\right)}{E\left(\frac{1}{\|X\|^{r}}\right)}\right) E\left(\frac{1}{\|X\|^{r}}\right) .
\end{aligned}
$$

Using Lemma 3.1, leads to

$$
\begin{aligned}
R_{\omega}\left(\delta_{a, r}, \theta\right) & \leq(1-\omega)\left\{p-2 a(p-r) E\left(\frac{1}{\|X\|^{r}}\right)\right\} \\
& +a^{2} 2^{-\frac{r-2}{2}} \frac{\Gamma\left(\frac{p-2 r+2}{2}\right)}{\Gamma\left(\frac{p-r}{2}\right)} E\left(\frac{1}{\|X\|^{r}}\right) \\
& =(1-\omega) p-2 a(1-\omega)(p-r) E\left(\frac{1}{\|X\|^{r}}\right) \\
& +2^{-\frac{r-2}{2}} a^{2} \frac{\Gamma\left(\frac{p-2 r+2}{2}\right)}{\Gamma\left(\frac{p-r}{2}\right)} E\left(\frac{1}{\|X\|^{r}}\right) .
\end{aligned}
$$


From the RHS of the last equality, it is easy to show that a sufficient condition for that $R_{\omega}\left(\delta_{a, r}, \theta\right) \leq R_{\omega}(X, \theta)=$ $(1-\omega) p$ and consequently $\delta_{a, r}$ dominates the MLE (so it is minimax), is

$$
-2 a(1-\omega)(p-r) E\left(\frac{1}{\|X\|^{r}}\right)+2^{-\frac{r-2}{2}} a^{2} \frac{\Gamma\left(\frac{p-2 r+2}{2}\right)}{\Gamma\left(\frac{p-r}{2}\right)} E\left(\frac{1}{\|X\|^{r}}\right) \leq 0,
$$

which is equivalent to

$$
2 a E\left(\frac{1}{\|X\|^{r}}\right)\left[-(1-\omega)(p-r)-2^{-\frac{r-2}{2}} a \frac{\Gamma\left(\frac{p-2 r+2}{2}\right)}{\Gamma\left(\frac{p-r}{2}\right)}\right] \leq 0,
$$

that leads to

$$
0 \leq a \leq 2^{\frac{r}{2}}(1-\omega)(p-r) \frac{\Gamma\left(\frac{p-r}{2}\right)}{\Gamma\left(\frac{p-2 r+2}{2}\right)} .
$$

ii) Using the convexity on $a$ of the function given in RHS of the equality (4), one can easily obtain the result. The proof of the Theorem is completed.

For $r=2$, we note $\widehat{a}$ by $d:=(1-\omega)(p-2)$, then we obtain the James-Stein estimator

$$
\delta_{J S}=\delta_{d, 2}=\left(1-d \frac{1}{\|X\|^{2}}\right) X .
$$

From Proposition 3.1 and Lemma 2.1, the risk function of $\delta_{J S}$ is

$$
R_{\omega}\left(\delta_{J S}, \theta\right)=(1-\omega) p-(p-2)^{2}(1-\omega)^{2} E\left(\frac{1}{p-2+2 K}\right)
$$

where $K \sim P\left(\frac{\|\theta\|^{2}}{2}\right)$.

From the formula (6), we note that

$$
R_{\omega}\left(\delta_{J S}, \theta\right) \leq(1-\omega) p=R_{\omega}(X, \theta),
$$

then $\delta_{J S}$ dominates the MLE $X$, therefore it is also minimax.

\subsection{Estimators dominating the James-Stein estimator}

Since the estimator $\delta_{a, r}=X-a \frac{1}{\|X\|^{r}} X$ dominates the MLE $X$ for certain values of $a$ and $r$, we think to add the term $b \frac{1}{\|X\|^{r}} X$ to the James-Stein estimator $\delta_{J S}$ to obtain an estimator that outperforms $\delta_{J S}$. Namely, we consider

$$
\delta_{b, r}=\delta_{J S}+b \frac{1}{\|X\|^{r}} X
$$

where $2 \leq r<\frac{p+2}{2}$ and the real positive constant $b$ may depend on $p$.

Proposition 3.2. Under the balanced squared error loss function $L_{\omega}$, the risk function of the estimator $\delta_{b, r}$ given in (7) is

$$
R_{\omega}\left(\delta_{b, r}, \theta\right)=R_{\omega}\left(\delta_{J S}, \theta\right)-2 b(1-\omega)(r-2) E\left(\frac{1}{\|X\|^{r}}\right)+b^{2} E\left(\frac{1}{\|X\|^{2 r-2}}\right)
$$


Proof. Using the risk function associated to the balanced loss function defined in (2), we obtain

$$
\begin{aligned}
R_{\omega}\left(\delta_{b, r}, \theta\right) & =\omega E\left(\left\|\delta_{J S}+b \frac{1}{\|X\|^{r}} X-X\right\|^{2}\right)+(1-\omega) E\left(\left\|\delta_{J S}+b \frac{1}{\|X\|^{r}} X-\theta\right\|^{2}\right) \\
& =\omega\left[E\left(\left\|\delta_{J S}-X\right\|^{2}\right)+E\left(\left\|b \frac{1}{\|X\|^{r}} X\right\|^{2}\right)+2 E\left(\left\langle\delta_{J S}-X, b \frac{1}{\|X\|^{r}} X\right\rangle\right)\right] \\
& +(1-\omega)\left[E\left(\left\|\delta_{J S}-\theta\right\|^{2}\right)+E\left(\left\|b \frac{1}{\|X\|^{r}} X\right\|^{2}\right)+2 E\left(\left\langle\delta_{J S}-\theta, b \frac{1}{\|X\|^{r}} X\right\rangle\right)\right] \\
& =R_{\omega}\left(\delta_{J S}, \theta\right)+b^{2} E\left(\frac{1}{\|X\|^{2 r-2}}\right)-2 \omega d b E\left(\frac{1}{\|X\|^{r}}\right) \\
& +2(1-\omega) E\left(\left\langle X-\theta-d \frac{1}{\|X\|^{2}} X, b \frac{1}{\|X\|^{r}} X\right\rangle\right) \\
& =R_{\omega}\left(\delta_{J S}, \theta\right)+b^{2} E\left(\frac{1}{\|X\|^{2 r-2}}\right)-2 \omega d b E\left(\frac{1}{\|X\|^{r}}\right)-2(1-\omega) d b E\left(\frac{1}{\|X\|^{r}}\right) \\
& +2(1-\omega) b \sum_{i=1}^{p} E\left[\left(X_{i}-\theta_{i}\right) \frac{X_{i}}{\|X\|^{r}}\right] \\
& =R_{\omega}\left(\delta_{J S}, \theta\right)+b^{2} E\left(\frac{1}{\|X\|^{2 r-2}}\right)-2 d b E\left(\frac{1}{\|X\|^{r}}\right) \\
& \left.+2(1-\omega) b \sum_{i=1}^{p} E\left(X_{i}-\theta_{i}\right) \frac{1}{\left(\|X\|^{2}\right)^{\frac{r}{2}}} X_{i}\right] .
\end{aligned}
$$

Using the Stein's Lemma 2.2, we get

$$
\begin{aligned}
R_{\omega}\left(\delta_{b, r}, \theta\right) & =R_{\omega}\left(\delta_{J S}, \theta\right)+b^{2} E\left(\frac{1}{\|X\|^{2 r-2}}\right)-2 d b E\left(\frac{1}{\|X\|^{r}}\right) \\
& +2(1-\omega) b \sum_{i=1}^{p} E\left[\frac{\partial}{\partial X_{i}}\left(\frac{1}{\|X\|^{2}} X_{i}\right)\right] \\
& =R_{\omega}\left(\delta_{J S}, \theta\right)+b^{2} E\left(\frac{1}{\|X\|^{2 r-2}}\right)-2 d b E\left(\frac{1}{\|X\|^{r}}\right) \\
& +2(1-\omega) b(p-r) E\left(\frac{1}{\|X\|^{r}}\right) \\
& =R_{\omega}\left(\delta_{J S}, \theta\right)+b^{2} E\left(\frac{1}{\|X\|^{2 r-2}}\right)+2 b[(1-\omega)(p-r)-d] E\left(\frac{1}{\|X\|^{r}}\right) \\
& =R_{\omega}\left(\delta_{J S}, \theta\right)+b^{2} E\left(\frac{1}{\|X\|^{2 r-2}}\right)-2 b(1-\omega)(r-2) E\left(\frac{1}{\|X\|^{r}}\right) .
\end{aligned}
$$

Theorem 3.3. Under the balanced squared error loss function $L_{\omega}$, the estimator $\delta_{b, r}$ with

$$
b=2^{\frac{r-2}{2}}(1-\omega)(r-2) \frac{\Gamma\left(\frac{p-r}{2}\right)}{\Gamma\left(\frac{p-2 r+2}{2}\right)},
$$

dominates the James-Stein estimator $\delta_{J S}$. 
Proof. By using Proposition 3.2, we have

$$
\begin{aligned}
R_{\omega}\left(\delta_{b, r}, \theta\right) & \leq R_{\omega}\left(\delta_{J S}, \theta\right)+b^{2} \frac{E\left(\frac{1}{\|X\|^{2 r-2}}\right)}{E\left(\frac{1}{\|X\|^{r}}\right)} E\left(\frac{1}{\|X\|^{r}}\right) \\
& -2 b(1-\omega)(r-2) b E\left(\frac{1}{\|X\|^{r}}\right) .
\end{aligned}
$$

By Lemma 3.1, we get

$$
\begin{aligned}
R_{\omega}\left(\delta_{b, r}, \theta\right) & \leq R_{\omega}\left(\delta_{J S}, \theta\right)+b^{2} 2^{-\frac{r-2}{2}} \frac{\Gamma\left(\frac{p-2 r+2}{2}\right)}{\Gamma\left(\frac{p-r}{2}\right)} E\left(\frac{1}{\|X\|^{r}}\right) \\
& -2 b(1-\omega)(r-2) b E\left(\frac{1}{\|X\|^{r}}\right) .
\end{aligned}
$$

The optimal value for $b$ that minimizes the RHS of the inequality (8), is

$$
\widehat{b}=2^{\frac{r-2}{2}}(1-\omega)(r-2) \frac{\Gamma\left(\frac{p-r}{2}\right)}{\Gamma\left(\frac{p-2 r+2}{2}\right)} .
$$

Thus

$$
\begin{aligned}
R_{\omega}\left(\delta_{\widehat{b}, r}, \theta\right) & \leq R_{\omega}\left(\delta_{J S}, \theta\right)-2^{\frac{r-2}{2}}(1-\omega)^{2}(r-2)^{2} \frac{\Gamma\left(\frac{p-r}{2}\right)}{\Gamma\left(\frac{p-2 r+2}{2}\right)} E\left(\frac{1}{\|X\|^{r}}\right) \\
& \leq R_{\omega}\left(\delta_{J S}, \theta\right) .
\end{aligned}
$$

\section{Simulation results}

We recall the form of the James-Stein estimator $\delta_{J S}$ given in (5)

$$
\delta_{J S}=\left(1-d \frac{1}{\|X\|^{2}}\right) X=\left(1-(1-\omega)(p-2) \frac{1}{\|X\|^{2}}\right) X
$$

where $d=(1-\omega)(p-2)$. Its risk function associated to the balanced squared error loss function $L_{\omega}$ is given by the formula (6). It is well known that the Positive-part of James-Stein estimator is defined by

$$
\delta_{J S}^{+}=\left(1-d \frac{1}{\|X\|^{2}}\right)^{+} X=\left(1-d \frac{1}{\|X\|^{2}}\right) X \mathrm{I}_{d \frac{1}{\|X\|^{2}} \leq 1}
$$

its risk function associated to $L_{\omega}$ is

$$
\begin{aligned}
R_{\omega}\left(\delta_{J S}^{+}, \theta\right) & =R_{\omega}\left(\delta_{J S}, \theta\right) \\
& +E\left[\left(\|X\|^{2}+d^{2} \frac{1}{\|X\|^{2}}-2(1-w) p\right) \mathrm{I}_{d \frac{1}{\|X\|^{2}} \geq 1}\right]
\end{aligned}
$$

with $\mathrm{I}_{d \frac{1}{\|X\|^{2}}} \geq 1$ denote the indicating function of the set $\left(d \frac{1}{\|X\|^{2}} \geq 1\right)$.

We also recall the estimator $\delta_{a, r}$ given in (3) where

$$
a=2^{\frac{r-2}{2}}(1-\omega)(p-r) \frac{\Gamma\left(\frac{p-r}{2}\right)}{\Gamma\left(\frac{p-2 r+2}{2}\right)}
$$


its risk function associated to $L_{\omega}$ is given in Proposition 3.1 and the estimator $\delta_{b, r}$ given in (7) where

$$
b=2^{\frac{r-2}{2}}(1-\omega)(r-2) \frac{\Gamma\left(\frac{p-r}{2}\right)}{\Gamma\left(\frac{p-2 r+2}{2}\right)},
$$

its risk function associated to $L_{\omega}$ is given in Proposition 3.2.

In this part, we firstly present the graphs of the risks ratios of the estimators $\delta_{J S}, \delta_{J S}^{+}, \delta_{a, r}$ and $\delta_{b, r}$, to the MLE $X$ denoted respectively: $\frac{R_{\omega}\left(\delta_{J S}, \theta\right)}{R_{\omega}(X, \theta)}, \frac{R_{\omega}\left(\delta_{J S}^{+}, \theta\right)}{R_{\omega}(X, \theta)}, \frac{R_{\omega}\left(\delta_{a, r}, \theta\right)}{R_{\omega}(X, \theta)}$ and $\frac{R_{\omega}\left(\delta_{b, r}, \theta\right)}{R_{\omega}(X, \theta)}$ as function of $\lambda=\frac{\|\theta\|^{2}}{2}$, for various values of $p, r$ and $\omega$. Secondly, we give tables that present the values of risks ratios $\frac{R_{\omega}\left(\delta_{J S}, \theta\right)}{R_{\omega}(X, \theta)}, \frac{R_{\omega}\left(\delta_{J S}^{+}, \theta\right)}{R_{\omega}(X, \theta)}, \frac{R_{\omega}\left(\delta_{a, r}, \theta\right)}{R_{\omega}(X, \theta)}$ and $\frac{R_{\omega}\left(\delta_{b, r}, \theta\right)}{R_{\omega}(X, \theta)}$ where in this case we fix $r$ and vary the values of $p$ and $\omega$.

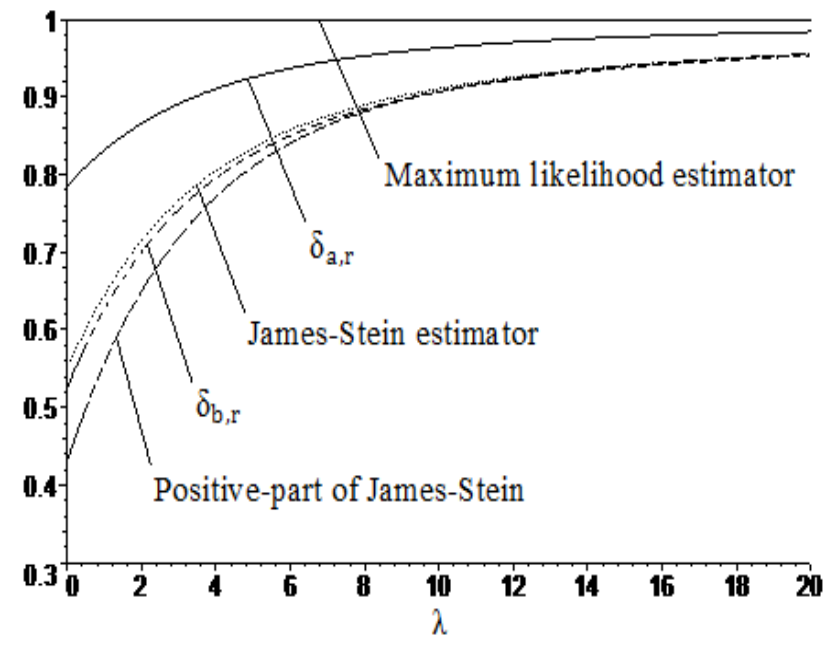

Figure 1: Graph of risks ratios $\frac{R_{\omega}\left(\delta_{J S}, \theta\right)}{R_{\omega}(X, \theta)}, \frac{R_{\omega}\left(\delta_{J S}^{+}, \theta\right)}{R_{\omega}(X, \theta)}, \frac{R_{\omega}\left(\delta_{a, r}, \theta\right)}{R_{\omega}(X, \theta)}$ and $\frac{R_{\omega}\left(\delta_{b, r}, \theta\right)}{R_{\omega}(X, \theta)}$ as functions of $\lambda=\frac{\|\theta\|^{2}}{2}$ for $p=4, r=$ 2.5 and $\omega=0.1$

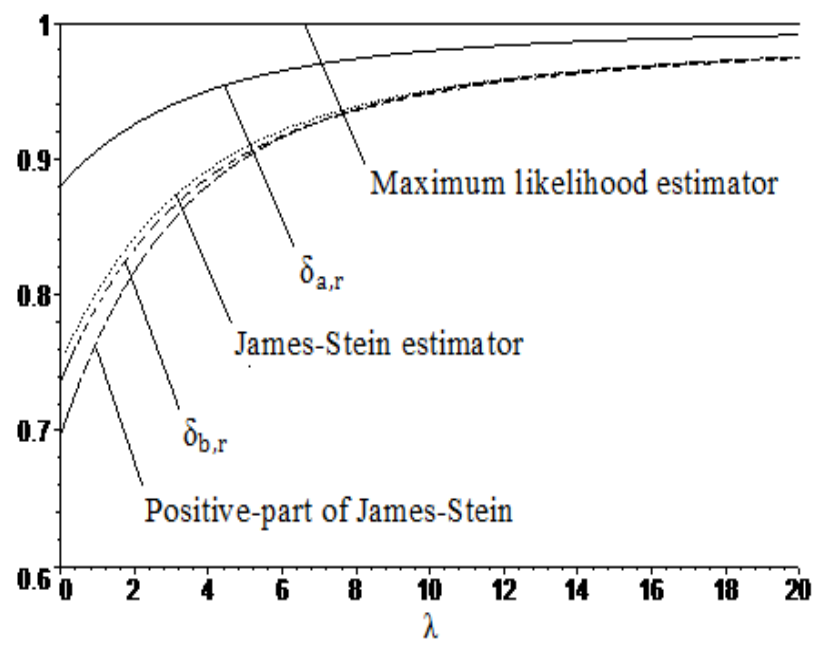

Figure 2: Graph of risks ratios $\frac{R_{\omega}\left(\delta_{J S}, \theta\right)}{R_{\omega}(X, \theta)}, \frac{R_{\omega}\left(\delta_{J S}^{+}, \theta\right)}{R_{\omega}(X, \theta)}, \frac{R_{\omega}\left(\delta_{a, r}, \theta\right)}{R_{\omega}(X, \theta)}$ and $\frac{R_{\omega}\left(\delta_{b, r}, \theta\right)}{R_{\omega}(X, \theta)}$ as functions of $\lambda=\frac{\|\theta\|^{2}}{2}$ for $p=4, r=$ 2.5 and $\omega=0.5$ 


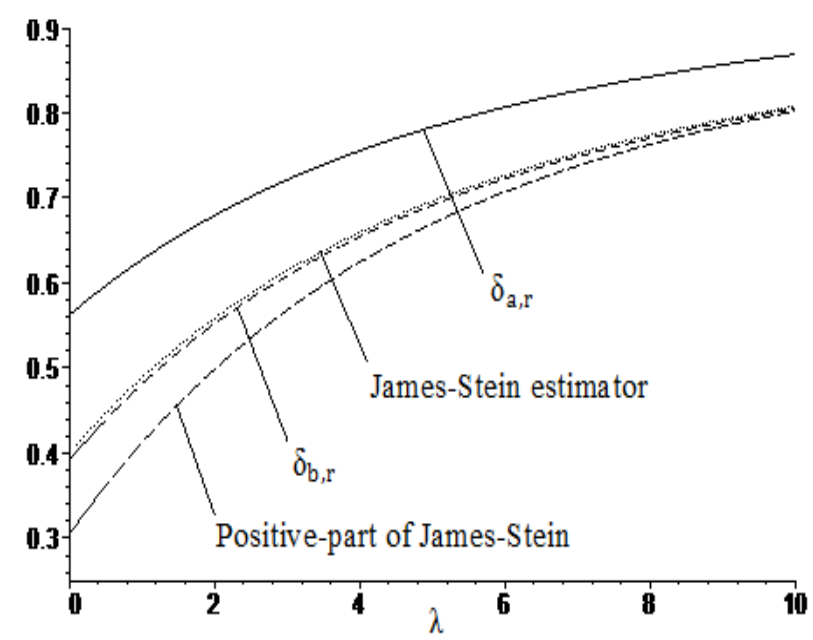

Figure 3: Graph of risks ratios $\frac{R_{\omega}\left(\delta_{J S}, \theta\right)}{R_{\omega}(X, \theta)}, \frac{R_{\omega}\left(\delta_{J S}^{+}, \theta\right)}{R_{\omega}(X, \theta)}, \frac{R_{\omega}\left(\delta_{a, r}, \theta\right)}{R_{\omega}(X, \theta)}$ and $\frac{R_{\omega}\left(\delta_{b, r}, \theta\right)}{R_{\omega}(X, \theta)}$ as functions of $\lambda=\frac{\|\theta\|^{2}}{2}$ for $p=6, r=$ 2.5 and $\omega=0.1$

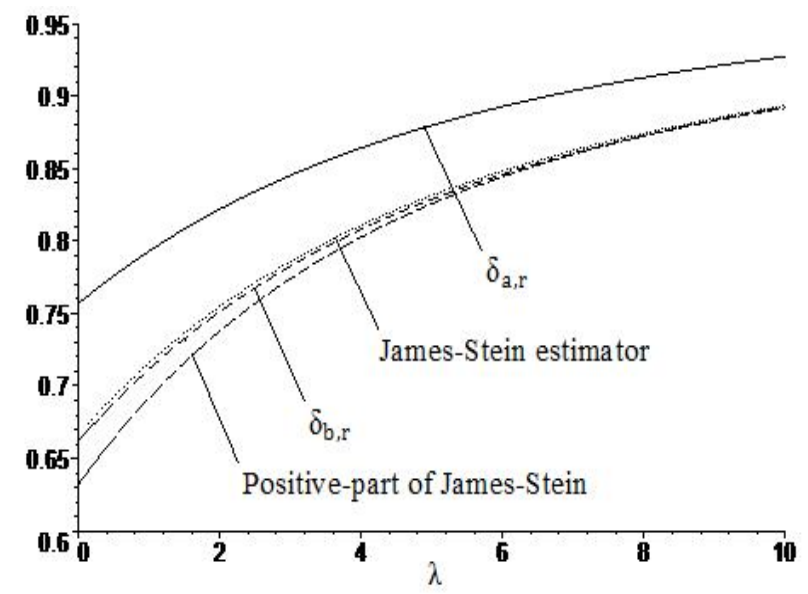

Figure 4: Graph of risks ratios $\frac{R_{\omega}\left(\delta_{J S}, \theta\right)}{R_{\omega}(X, \theta)}, \frac{R_{\omega}\left(\delta_{J S}^{+}, \theta\right)}{R_{\omega}(X, \theta)}, \frac{R_{\omega}\left(\delta_{a, r}, \theta\right)}{R_{\omega}(X, \theta)}$ and $\frac{R_{\omega}\left(\delta_{b, r}, \theta\right)}{R_{\omega}(X, \theta)}$ as functions of $\lambda=\frac{\|\theta\|^{2}}{2}$ for $p=6, r=$ 2.5 and $\omega=0.5$ 


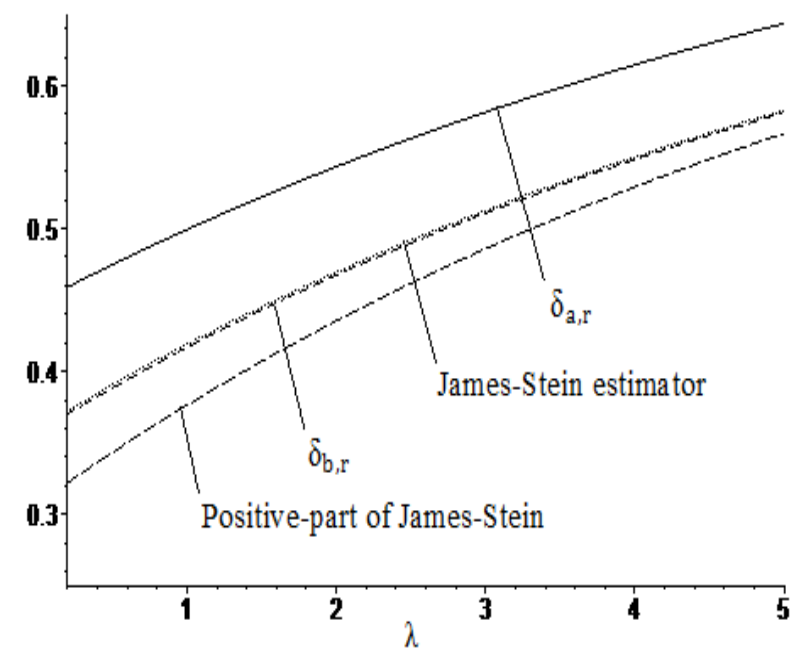

Figure 5: Graph of risks ratios $\frac{R_{\omega}\left(\delta_{J S}, \theta\right)}{R_{\omega}(X, \theta)}, \frac{R_{\omega}\left(\delta_{J S}^{+}, \theta\right)}{R_{\omega}(X, \theta)}, \frac{R_{\omega}\left(\delta_{a, r}, \theta\right)}{R_{\omega}(X, \theta)}$ and $\frac{R_{\omega}\left(\delta_{b, r}, \theta\right)}{R_{\omega}(X, \theta)}$ as functions of $\lambda=\frac{\|\theta\|^{2}}{2}$ for $p=$ $10, r=2.5$ and $\omega=0.2$

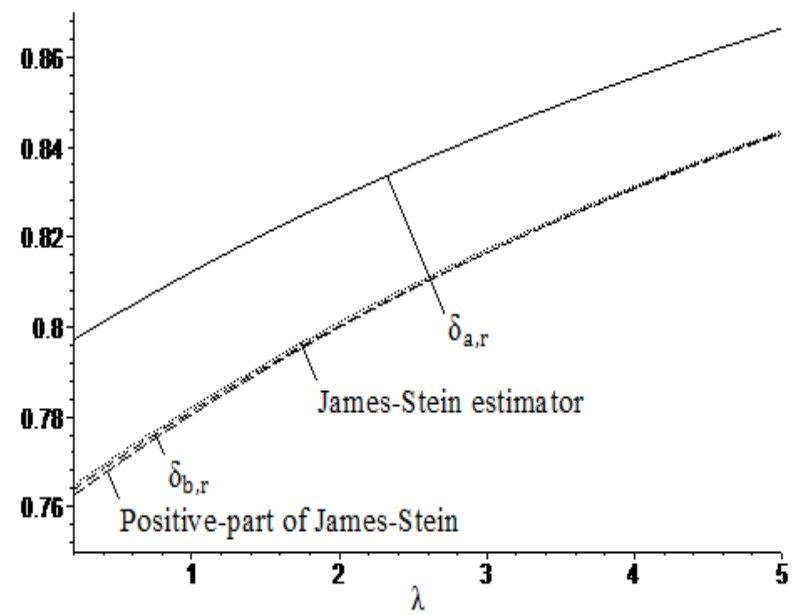

Figure 6: Graph of risks ratios $\frac{R_{\omega}\left(\delta_{J S}, \theta\right)}{R_{\omega}(X, \theta)}, \frac{R_{\omega}\left(\delta_{J S}^{+}, \theta\right)}{R_{\omega}(X, \theta)}, \frac{R_{\omega}\left(\delta_{a, r}, \theta\right)}{R_{\omega}(X, \theta)}$ and $\frac{R_{\omega}\left(\delta_{b, r}, \theta\right)}{R_{\omega}(X, \theta)}$ as functions of $\lambda=\frac{\|\theta\|^{2}}{2}$ for $p=$ $10, r=2.5$ and $\omega=0.7$ 
The figures 1-6 show that the risks ratios $\frac{R_{\omega}\left(\delta_{J S}, \theta\right)}{R_{\omega}(X, \theta)}, \frac{R_{\omega}\left(\delta_{J S}^{+}, \theta\right)}{R_{\omega}(X, \theta)}, \frac{R_{\omega}\left(\delta_{a, r}, \theta\right)}{R_{\omega}(X, \theta)}$ and $\frac{R_{\omega}\left(\delta_{b, r}, \theta\right)}{R_{\omega}(X, \theta)}$ are less than 1 , then the estimators $\delta_{J S}, \delta_{J S}^{+}, \delta_{a, r}$ and $\delta_{b, r}$ dominate the MLE $X$ for diverse values of $p, r$ and $\omega$, therefore are minimax. We note that the estimator $\delta_{b, r}$ dominates the James-Stein estimator $\delta_{J S}$. We also observe that the gain increases if $\omega$ is near to 0 and decreases if $\omega$ is near to 1 . The following tables illustrate this note. In these tables we give the values of the risks ratios $\frac{R_{\omega}\left(\delta_{J S}, \theta\right)}{R_{\omega}(X, \theta)}, \frac{R_{\omega}\left(\delta_{J S}^{+}, \theta\right)}{R_{\omega}(X, \theta)}, \frac{R_{\omega}\left(\delta_{a, r}, \theta\right)}{R_{\omega}(X, \theta)}$ and $\frac{R_{\omega}\left(\delta_{b, r}, \theta\right)}{R_{\omega}(X, \theta)}$ for the different values of $p, \omega$ and $\lambda$ when $r=2.5$. The first entry is $\frac{R_{\omega}\left(\delta_{a, r}, \theta\right)}{R_{\omega}(X, \theta)}$, the second entry is $\frac{R_{\omega}\left(\delta_{J S}, \theta\right)}{R_{\omega}(X, \theta)}$, the third entry is $\frac{R_{\omega}\left(\delta_{b, r}, \theta\right)}{R_{\omega}(X, \theta)}$ and the fourth entry is $\frac{R_{\omega}\left(\delta_{J S}^{+}, \theta\right)}{R_{\omega}(X, \theta)}$.

Table 1: $p=4$ and $r=2.5$

\begin{tabular}{|c|c|c|c|c|c|c|}
\hline$\lambda$ & $\omega=0.0$ & $\omega=0.1$ & $\omega=0.2$ & $\omega=0.5$ & $\omega=0.7$ & $\omega=0.9$ \\
\hline \multirow[b]{4}{*}{0.4359} & 0.7860 & 0.8074 & 0.8288 & 0.8930 & 0.9358 & 0.9786 \\
\hline & 0.5507 & 0.5957 & 0.6406 & 0.7754 & 0.8652 & 0.9551 \\
\hline & 0.5270 & 0.5743 & 0.6216 & 0.7635 & 0.8581 & 0.9527 \\
\hline & 0.4326 & 0.4913 & 0.5508 & 0.7303 & 0.8462 & 0.9526 \\
\hline \multirow[b]{4}{*}{1.2418} & 0.8232 & 0.8408 & 0.8585 & 0.9116 & 0.9469 & 0.9823 \\
\hline & 0.6275 & 0.6648 & 0.7020 & 0.8138 & 0.8882 & 0.9627 \\
\hline & 0.6079 & 0.6471 & 0.6863 & 0.8039 & 0.8824 & 0.9608 \\
\hline & 0.5326 & 0.5826 & 0.6326 & 0.7809 & 0.8748 & 0.9611 \\
\hline \multirow[b]{4}{*}{3.7523} & 0.8957 & 0.9061 & 0.9166 & 0.9479 & 0.9687 & 0.9896 \\
\hline & 0.7743 & 0.7969 & 0.8194 & 0.8871 & 0.9323 & 0.9774 \\
\hline & 0.7627 & 0.7865 & 0.8102 & 0.8814 & 0.9288 & 0.9763 \\
\hline & 0.7298 & 0.7601 & 0.7898 & 0.8750 & 0.9278 & 0.9769 \\
\hline \multirow[b]{4}{*}{5.0019} & 0.9169 & 0.9252 & 0.9335 & 0.9584 & 0.9751 & 0.9917 \\
\hline & 0.8165 & 0.8348 & 0.8532 & 0.9082 & 0.9449 & 0.9816 \\
\hline & 0.8072 & 0.8265 & 0.8458 & 0.9036 & 0.9422 & 0.9807 \\
\hline & 0.7868 & 0.8108 & 0.8342 & 0.9009 & 0.9423 & 0.9814 \\
\hline \multirow[b]{4}{*}{10.4311} & 0.9608 & 0.9648 & 0.9687 & 0.9804 & 0.9882 & 0.9961 \\
\hline & 0.9046 & 0.9142 & 0.9237 & 0.9523 & 0.9714 & 0.9905 \\
\hline & 0.9003 & 0.9103 & 0.9202 & 0.9501 & 0.9700 & 0.9900 \\
\hline & 0.9002 & 0.9108 & 0.9212 & 0.9515 & 0.9712 & 0.9904 \\
\hline \multirow[b]{4}{*}{15.4110} & 0.9753 & 0.9778 & 0.9802 & 0.9876 & 0.9926 & 0.9975 \\
\hline & 0.9351 & 0.9416 & 0.9481 & 0.9676 & 0.9805 & 0.9935 \\
\hline & 0.9324 & 0.9391 & 0.9459 & 0.9662 & 0.9797 & 0.9932 \\
\hline & 0.9344 & 0.9411 & 0.9477 & 0.9678 & 0.9805 & 0.9935 \\
\hline \multirow[b]{4}{*}{20.0000} & 0.9820 & 0.9838 & 0.9856 & 0.9910 & 0.9946 & 0.9982 \\
\hline & 0.9500 & 0.9550 & 0.9600 & 0.9750 & 0.9850 & 0.9950 \\
\hline & 0.9480 & 0.9532 & 0.9584 & 0.9740 & 0.9844 & 0.9948 \\
\hline & 0.9499 & 0.9549 & 0.9599 & 0.9750 & 0.9850 & 0.9950 \\
\hline
\end{tabular}


Table 2: $p=8$ and $r=2.5$

\begin{tabular}{|c|c|c|c|c|c|c|}
\hline$\lambda$ & $\omega=0.0$ & $\omega=0.1$ & $\omega=0.2$ & $\omega=0.5$ & $\omega=0.7$ & $\omega=0.9$ \\
\hline & 0.4189 & 0.4770 & 0.5351 & 0.7094 & 0.8257 & 0.9419 \\
& 0.2891 & 0.3602 & 0.4313 & 0.6446 & 0.7867 & 0.9289 \\
& 0.2843 & 0.3559 & 0.4275 & 0.6422 & 0.7853 & 0.9284 \\
\hline \multirow{5}{*}{1.4359} & 0.2059 & 0.2884 & 0.3727 & 0.6252 & 0.7826 & 0.9288 \\
\hline & 0.4719 & 0.5247 & 0.5775 & 0.7359 & 0.8416 & 0.9472 \\
& 0.3533 & 0.4180 & 0.4827 & 0.6767 & 0.8060 & 0.9353 \\
& 0.3490 & 0.4141 & 0.4792 & 0.6745 & 0.8047 & 0.9349 \\
& 0.2800 & 0.3567 & 0.4341 & 0.6619 & 0.8030 & 0.9353 \\
\hline & 0.8957 & 0.9061 & 0.9166 & 0.9479 & 0.9687 & 0.9896 \\
& 0.7743 & 0.7969 & 0.8194 & 0.8871 & 0.9323 & 0.9774 \\
& 0.7627 & 0.7865 & 0.8102 & 0.8814 & 0.9288 & 0.9763 \\
& 0.7298 & 0.7601 & 0.7898 & 0.8750 & 0.9278 & 0.9769 \\
\hline \multirow{5}{*}{5.0019} & 0.6419 & 0.6777 & 0.7135 & 0.8209 & 0.8926 & 0.9642 \\
& 0.5557 & 0.6001 & 0.6446 & 0.7778 & 0.8667 & 0.9556 \\
& 0.5527 & 0.5975 & 0.6422 & 0.7764 & 0.8658 & 0.9553 \\
& 0.5221 & 0.5749 & 0.6266 & 0.7739 & 0.8661 & 0.9556 \\
\hline \multirow{3}{*}{0.4311} & 0.7673 & 0.7905 & 0.8138 & 0.8836 & 0.9302 & 0.9767 \\
& 0.7025 & 0.7322 & 0.7620 & 0.8512 & 0.9107 & 0.9702 \\
& 0.7005 & 0.7305 & 0.7604 & 0.8503 & 0.9102 & 0.9700 \\
& 0.6944 & 0.7267 & 0.7585 & 0.8507 & 0.9107 & 0.9702 \\
\hline & 0.8282 & 0.8454 & 0.8626 & 0.9141 & 0.9485 & 0.9828 \\
& 0.7739 & 0.7966 & 0.8192 & 0.8870 & 0.9322 & 0.9774 \\
& 0.7725 & 0.7953 & 0.8180 & 0.8863 & 0.9318 & 0.9772 \\
15.4110 & 0.7721 & 0.7954 & 0.8185 & 0.8869 & 0.9322 & 0.9774 \\
\hline & 0.8632 & 0.8769 & 0.8906 & 0.9316 & 0.9590 & 0.9863 \\
& 0.8155 & 0.8339 & 0.8524 & 0.9077 & 0.9446 & 0.9815 \\
& 0.8144 & 0.8329 & 0.8515 & 0.9072 & 0.9443 & 0.9814 \\
& 0.8150 & 0.8337 & 0.8522 & 0.9077 & 0.9446 & 0.9815 \\
\hline
\end{tabular}

From the results presented in Tables 1 and 2, we see that if $\omega$ and $\lambda=\frac{\|\theta\|^{2}}{2}$ are small, the gain of the risks ratios $\frac{R_{\omega}\left(\delta_{J S}, \theta\right)}{R_{\omega}(X, \theta)}, \frac{R_{\omega}\left(\delta_{J S}^{+}, \theta\right)}{R_{\omega}(X, \theta)}, \frac{R_{\omega}\left(\delta_{a, r}, \theta\right)}{R_{\omega}(X, \theta)}$ and $\frac{R_{\omega}\left(\delta_{b, r}, \theta\right)}{R_{\omega}(X, \theta)}$ is very important. Also, if the values of $\omega$ and $\lambda$ increase, the gain decreases and approaches zero, a little improvement is then obtained. We also observe that, if the values of $p$ increase, the gain increases and this for each fixed value of $\omega$. We also see that, if the values of $p$ are large, the gain is large and consequently we obtain more improvement. We conclude that, the gain is important when the parameters $p$ and $\lambda$ are large and $\omega$ is near to 0 . As seen above, the gain of the risks ratios is influenced by various values of $\omega, p$ and $\lambda$.

We also remark that if $\lambda$ increases or $\omega$ is near to 1 , the estimator $\delta_{b, r}$ not only dominates $\delta_{J S}$ but also dominates the estimator $\delta_{J S}^{+}$. We will give the following figures (figures 7-8) that confirm this note. 


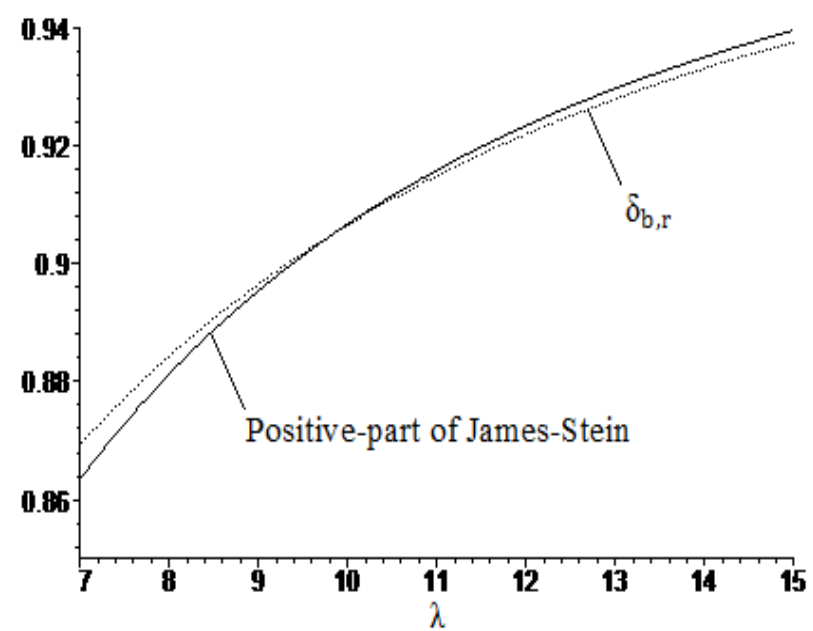

Figure 7: Graph of risks ratios $\frac{R_{\omega}\left(\delta_{J S}^{+}, \theta\right)}{R_{\omega}(X, \theta)}$ and $\frac{R_{\omega}\left(\delta_{b, r}, \theta\right)}{R_{\omega}(X, \theta)}$ as functions of $\lambda=\frac{\|\theta\|^{2}}{2}$ for $p=4, r=2.5$ and $\omega=0.1$

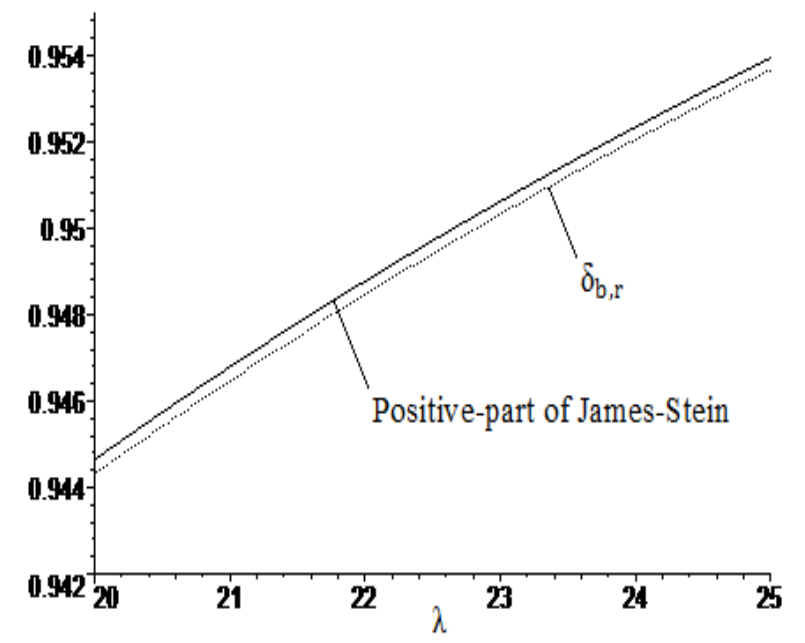

Figure 8: Graph of risks ratios $\frac{R_{\omega}\left(\delta_{J S}^{+}, \theta\right)}{R_{\omega}(X, \theta)}$ and $\frac{R_{\omega}\left(\delta_{b, r}, \theta\right)}{R_{\omega}(X, \theta)}$ as functions of $\lambda=\frac{\|\theta\|^{2}}{2}$ for $p=8, r=2.5$ and $\omega=0.7$

\section{Appendix}

Proof. (Proof of Lemma 3.1) First, we show that, for any real $v$

$$
\frac{\partial}{\partial \lambda} E\left(U^{v}\right)=\frac{\partial}{\partial \lambda} \int_{R_{+}} x^{v} \chi_{p}^{2}(\lambda ; d x)=v 2^{v-1} \sum_{k=0}^{+\infty} \frac{\Gamma\left(\frac{p}{2}+v+k\right)}{\Gamma\left(\frac{p}{2}+1+k\right)} P\left(\frac{\lambda}{2} ; d k\right)
$$

where $P\left(\frac{\lambda}{2}\right)$ being the Poisson distribution of parameter $\frac{\lambda}{2}$.

Using the formula (1) we have, for any real $v$

$$
E\left(U^{v}\right)=E\left[\left(\chi_{p}^{2}(\lambda)\right)^{v}\right]=E\left[\left(\chi_{p+2 K}^{2}\right)^{v}\right]=2^{v} E\left[\frac{\Gamma\left(\frac{p}{2}+K+v\right)}{\Gamma\left(\frac{p}{2}+K\right)}\right],
$$

where $K \sim P\left(\frac{\lambda}{2}\right)$. Then

$$
\frac{\partial}{\partial \lambda} E\left(U^{v}\right)=\frac{\partial}{\partial \lambda} \int_{R_{+}} x^{v} \chi_{p}^{2}(\lambda ; d x)
$$




$$
\begin{aligned}
& =2^{v} \sum_{k=0}^{+\infty}\left[\frac{\Gamma\left(\frac{p}{2}+k+v\right)}{\Gamma\left(\frac{p}{2}+k\right)}\right] \frac{1}{k !} \frac{\partial}{\partial \lambda}\left[\left(\frac{\lambda}{2}\right)^{k} \exp \left(-\frac{\lambda}{2}\right)\right] \\
& =2^{v-1} \sum_{k=0}^{+\infty}\left[\frac{\Gamma\left(\frac{p}{2}+k+v\right)}{\Gamma\left(\frac{p}{2}+k\right)}\right] \frac{1}{k !} \exp \left(-\frac{\lambda}{2}\right)\left[-\left(\frac{\lambda}{2}\right)^{k}+k\left(\frac{\lambda}{2}\right)^{k-1}\right] \\
& =2^{v-1} \exp \left(-\frac{\lambda}{2}\right)\left\{-\sum_{k=0}^{+\infty}\left[\frac{\Gamma\left(\frac{p}{2}+k+v\right)}{\Gamma\left(\frac{p}{2}+k\right)}\right] \frac{1}{k !}\left(\frac{\lambda}{2}\right)^{k}\right\} \\
& +2^{v-1} \exp \left(-\frac{\lambda}{2}\right)\left\{\sum_{k=0}^{+\infty}\left[\frac{\Gamma\left(\frac{p}{2}+k+v+1\right)}{\Gamma\left(\frac{p}{2}+k+1\right)}\right] \frac{1}{k !}\left(\frac{\lambda}{2}\right)^{k}\right\} \\
& =2^{v-1} \exp \left(-\frac{\lambda}{2}\right)\left\{\sum_{k=0}^{+\infty} \frac{1}{k !}\left(\frac{\lambda}{2}\right)^{k}\left[\frac{\Gamma\left(\frac{p}{2}+k+v\right)}{\Gamma\left(\frac{p}{2}+k+1\right)}\right]\left[-\left(\frac{p}{2}+k\right)+\left(\frac{p}{2}+v+k\right)\right]\right\} \\
& =v 2^{v-1} \sum_{k=0}^{+\infty} \frac{\Gamma\left(\frac{p}{2}+v+k\right)}{\Gamma\left(\frac{p}{2}+1+k\right)} P\left(\frac{\lambda}{2} ; d k\right) .
\end{aligned}
$$

Let the function

$$
\begin{aligned}
K_{p, r, s}(\lambda) & =\left(\frac{\partial}{\partial \lambda} \int_{R_{+}} x^{r} \chi_{p}^{2}(\lambda ; d x)\right)\left(\int_{R_{+}} x^{s} \chi_{p}^{2}(\lambda ; d x)\right) \\
& -\left(\frac{\partial}{\partial \lambda} \int_{R_{+}} x^{s} \chi_{p}^{2}(\lambda ; d x)\right)\left(\int_{R_{+}} x^{r} \chi_{p}^{2}(\lambda ; d x)\right) .
\end{aligned}
$$

For the function $H_{p, r, s}$ to be strictly increasing, it suffices that the function $K_{p, r, s}$ takes positive values. From the equality (9), we obtain

$$
\begin{aligned}
K_{p, r, s}(\lambda) & =2^{r+s-1} r \sum_{i=0}^{+\infty} \sum_{j=0}^{+\infty} \frac{\Gamma\left(\frac{p}{2}+r+i\right)}{\Gamma\left(\frac{p}{2}+i+1\right)} \frac{\Gamma\left(\frac{p}{2}+s+j\right)}{\Gamma\left(\frac{p}{2}+j\right)} P\left(\frac{\lambda}{2} ; d i\right) P\left(\frac{\lambda}{2} ; d j\right) \\
& -2^{r+s-1} s \sum_{i=0}^{+\infty} \sum_{j=0}^{+\infty} \frac{\Gamma\left(\frac{p}{2}+r+j\right)}{\Gamma\left(\frac{p}{2}+j\right)} \frac{\Gamma\left(\frac{p}{2}+s+i\right)}{\Gamma\left(\frac{p}{2}+i+1\right)} P\left(\frac{\lambda}{2} ; d j\right) P\left(\frac{\lambda}{2} ; d i\right)
\end{aligned}
$$

As, $r>s$ then

$$
K_{p, r, s}(\lambda) \geq r 2^{r+s-1} \sum_{i=0}^{+\infty} \sum_{j=0}^{+\infty} l_{p, r, s}(i, j) P\left(\frac{\lambda}{2} ; d i\right) P\left(\frac{\lambda}{2} ; d j\right)
$$

where

$$
l_{p, r, s}(i, j)=\frac{\Gamma\left(\frac{p}{2}+r+i\right) \Gamma\left(\frac{p}{2}+s+j\right)-\Gamma\left(\frac{p}{2}+r+j\right) \Gamma\left(\frac{p}{2}+s+i\right)}{\Gamma\left(\frac{p}{2}+i+1\right) \Gamma\left(\frac{p}{2}+j\right)} .
$$

We note that, for any $i, l_{p, r, s}(i, j)=0$; then we have

$$
K_{p, r, s}(i, j) \geq r 2^{r+s-1} \sum_{i=0}^{+\infty} \sum_{j>i}^{+\infty}\left(l_{p, r, s}(i, j)+l_{p, r, s}(j, i)\right) P\left(\frac{\lambda}{2} ; d i\right) P\left(\frac{\lambda}{2} ; d j\right) .
$$


But if $i<j$, we get

$$
\begin{aligned}
l_{p, r, s}(i, j)+l_{p, r, s}(j, i) & =\left(\Gamma\left(\frac{p}{2}+r+i\right) \Gamma\left(\frac{p}{2}+s+j\right)-\Gamma\left(\frac{p}{2}+r+j\right) \Gamma\left(\frac{p}{2}+s+i\right)\right) \\
& \times\left[\frac{1}{\Gamma\left(\frac{p}{2}+i+1\right) \Gamma\left(\frac{p}{2}+j\right)}-\frac{1}{\Gamma\left(\frac{p}{2}+j+1\right) \Gamma\left(\frac{p}{2}+i\right)}\right] \\
& =\frac{\Gamma\left(\frac{p}{2}+r+i\right) \Gamma\left(\frac{p}{2}+s+i\right)}{\Gamma\left(\frac{p}{2}+i\right) \Gamma\left(\frac{p}{2}+j\right)}\left[\frac{1}{\frac{p}{2}+i}-\frac{1}{\frac{p}{2}+j}\right] \\
& \times\left[\prod_{t=0}^{j-i-1}\left(\frac{p}{2}+s+i+t\right)-\prod_{t=0}^{j+i-1}\left(\frac{p}{2}+r+i+t\right)\right] \\
& \leq 0
\end{aligned}
$$

because for any $t, \frac{p}{2}+s+i+t<\frac{p}{2}+r+i+t$. As in hypothesis $r<0$, we have $K_{p, r, s}(\lambda)>0$. Thus, we obtain the desired result.

ii) Using i) it is clear that the function $H_{p, r}^{1}(\lambda)=\frac{E\left(\|X\|^{-r}\right)}{E\left(\|X\|^{-2 r+2}\right)}$ is non-decreasing on $\lambda$, then the function $\frac{1}{H_{p, r}^{1}(\lambda)}$ is non-increasing on $\lambda$, thus

$$
\begin{aligned}
\sup _{\|\theta\|}\left(\frac{E\left(\|X\|^{-2 r+2}\right)}{E\left(\|X\|^{-r}\right)}\right) & =\sup _{\|\theta\|}\left(\frac{1}{H_{p, r}^{1}(\lambda)}\right) \\
& =\frac{1}{H_{p, r}^{1}(0)} \\
& =2^{\frac{-r+2}{2}} \frac{\Gamma\left(\frac{p}{2}-r+1\right)}{\Gamma\left(\frac{p-r}{2}\right)}
\end{aligned}
$$

\section{Conclusion}

In this work, we studied the estimating of the unknown mean $\theta$ of a multivariate normal distribution $X \sim N_{p}\left(\theta, \sigma^{2} I_{p}\right)$ where $\sigma^{2}$ is known using the risk associated to the balanced loss function as a tool to compare two estimators. First, we established the minimaxity of the estimators defined by $\delta_{a, r}=\left(1-a\left(1 /\|X\|^{r}\right)\right) X$, where $2 \leq r<(p+2) / 2$ and the real constant $a$ may depend on $p$. Secondly, we showed that the estimator $\delta_{b, r}=\delta_{J S}+b\left(1 /\|X\|^{r}\right) X$ with $2 \leq r<(p+2) / 2$ and the real constant $b$ may depend on $p$, dominates the James-Stein estimator $\delta_{J S}$, thus it is also minimax. In the simulation results we noted that if $\lambda=\|\theta\|^{2} / 2$ increases, the estimator $\delta_{b, r}$ not only dominates $\delta_{J S}$ but also dominates the estimator $\delta_{J S}^{+}$. An attempt will be made to construct a subclass of the estimators $\delta_{b, r}$ which dominate the positive-part of James-Stein $\delta_{J S}^{+}$for all values of $\lambda$ and treat the behaviour of risks ratios of our considered estimators to the MLE, when the dimension of parameters space $p$ tends to infinity. The study will serve the purpose to extend this work for a model having a symmetrical spherical distribution.

\section{Acknowledgement}

The authors would like to thank the editor and the referees for their comments and insightful suggestions, and careful reading of the manuscript. This research was partially supported by the DGRSDT-MESRS-Algeria.

\section{References}

1. Amin, M., Nauman, A. M., and Amanullah, M. (2020). On the james-stein estimator for the poisson regression model. Comm. Statist. Simulation Comput., pages 1-14, doi.org/10.1080/03610918.2020.1775851.

2. Arnold, F. S. (1981). The theory of Linear models and Multivariate analysis, pp. 9-10. John Wiley \& Sons, New York, USA.

3. Benkhaled, A. and Hamdaoui, A. (2019). General classes of shrinkage estimators for the multivariate normal mean with unknown variancee: Minimaxity and limit of risks ratios. Kragujevac J. Math., 46:193-213. 
4. Guikai, H., Qingguo, L., and Shenghua, L. (2014). Trisk comparison of improved estimators in a linear regression model with multivariate t errors under balanced loss function. Journal of Applied Mathematics, 354:1-7, doi.org/10.1155/2014/129205.

5. Hamdaoui, A., Benkhaled, A., and Mezouar, N. (2020). Minimaxity and limits of risks ratios of shrinkage estimators of a multivariate normal mean in the bayesian case. Stat. Optim. Inf. Comput., 8:507-520, doi.org/10.19139/soic-2310-5070-735.

6. Jafari, J., Leblan, A., and Marchand, E. (2014). On continuous distribution functions, minimax and bes invariant estimators and integrated balanced loss functions. Canad. J. Statistist., 42:470-486, doi.org/10.1002/cjs.11217.

7. James, W. and Stein, C. (1961). Estimation with quadratic loss. Fourth Berkeley Symp. on Math. Statist. and Prob., Univ. of Calif. Press, Berkeley, 1:361-379.

8. Karamikabir, H., Afsahri, M., and Arashi, M. (2018). Shrinkage estimation of non-negative mean vector with unknown covariance under balance loss. Journal of Inequalities and Applications, pages 1-11, doi.org/10.1186/s13660-018-1919-0.

9. Sanjari, F. and Asgharzadeh, A. (2004). Estimation of a normal mean relative to balanced loss functions. Statistical Papers, 45:279-286, doi.org/10.1007/BF02777228.

10. Selahattin, K. and Issam, D. (2019). The optimal extended balanced loss function estimators. J. Comput. Appl. Math., 345:86-98, doi.org/10.1016/j.cam.2018.06.021.

11. Selahattin, K., Sadullah, S., özkale, M. R., and Güler, H. (2011). More on the restricted ridge regression estimation. J. Stat. Comput. Simul., 81:1433-1448, doi.org/10.1080/00949655.2010.491480.

12. Stein, C. (1956). Inadmissibilty of the usual estimator for the mean of a multivariate normal distribution. Proc. 3th Berkeley Symp. on Math. Statist. and Prob., Univ. of Calif. Press, Berkeley, 1:197-206.

13. Stein, C. (1981). Estimation of the mean of a multivariate normal distribution. International Statistical Review, 9:1135-1151, doi.org/10.1214/aos/1176345632.

14. Tsukuma, H. and Kubukawa, T. (2015). Estimation of the mean vector in a singular multivariate normal distribution. J. Multivariate Anal., 140:215-232, doi.org/10.1016/j.jmva.2015.05.016.

15. Xie, X., Kou, S. C., and Brown, B. (2016). Optimal shrinkage estimators of mean parameters in family of distribution with quadratic variance. Ann. Statist., 44:564-597, doi.org/10.1214/15-AOS1377.

16. Yuzba, B., Arashi, M., and Ahmed, S. (2020). Shrinkage estimation strategies in generalised ridge regression models: Low/high-dimension regime. pages 1-23, doi.org/10.1111/insr.12351. Int. Stat. Rev.

17. Zellner, A. (1994). Bayesian and non-bayesian estimation using balanced loss functions. Statistical Decision Theory and Related Topics, 8:337-390.

18. Zinodiny, S., Leblan, S., and Nadarajah, S. (2017). Bayes minimax estimation of the mean matrix of matrix-variate normal distribution under balanced loss function. Statist. Probab. Lett., 125:110-120, doi.org/10.1016/j.spl.2017.02.003. 